\title{
An Extensor Digitorum Muscle for Index Finger Originated from the Extensor Carpi Radialis Brevis
}

\author{
Jongwan Kim $\mathbb{D}^{1}$, Jae-Ho Lee $\mathbb{D}^{1, *}$ \\ ${ }^{1}$ Department of Anatomy, School of Medicine, Keimyung University, 1095 Dalgubeol-daero, Daegu 42601, Republic of Korea
}

\begin{abstract}
The anomalous variations in the extensor carpi radialis brevis (ECRB) and extensor digitorum (ED) muscles of the forearm have been previously reported in the literature. The present article reported an anomalous variation of the ECRB and ED muscles in the forearm. We found an accessory muscle originated from the medial side of the ECRB muscle and inserted into the base of second metacarpal bone. And the ED muscle divides into three distinct tendons without the tendon of index finger. This case is a first report of the variations of ECRB and ED muscle in Korean cadaver. Knowledge of this variation is important to avoid surgical complications and to prevent diagnostic problems. Its embryological and clinical significances were discussed.
\end{abstract}

Keywords : Anatomical variations, Accessory muscle, Extensor carpi radialis brevis, Extensor digitorum

\section{INTRODUCTION}

The extensor carpi radialis brevis (ECRB) muscle arises from the lateral epicondyle of the humerus and the belly of the ECRB muscle end is a flat tendon and inserts onto the dorsal surface of the base of the third metacarpal. The extensor digitorum (ED) muscle originates from the lateral epicondyle of the humerus and it divides into four tendons. Each of these tendons inserts lateral and dorsal surface of phalanges of index, middle, ring, and little fingers.

The anomalous variations of these muscles of the forearm have been reported in previous study [1]. The extensor tendons of the fingers have a variable arrangement on

The author(s) agree to abide by the good publication practice guideline for medical journals.

The author(s) declare that there are no conflicts of interest.

Received: August 16, 2019; Revised: October 7, 2019;

Accepted: October 14, 2019

Correspondence to: Jae-Ho Lee (Department of Anatomy, Keimyung University School of Medicine, 2800, Dalgubeoldaero, Dalseo-Gu, Daegu, Republic of Korea)

E-mail: anato82@dsmc.or.kr dorsal aspect of hand. When transplantation of extensor tendons is undertaken, it can result in confusion, unless the surgeon is completely aware of possible arrangements of the tendons, as well as the relative frequency of the variations $[1,2]$. Knowledge of the extensor tendons is significantly value in planning tendon graft surgeries $[1,3]$. Successful tenosynovectomy in the treatment of de Quervain's disease requires an adequate anatomical knowledge of extensor tendons $[1,4]$. The tendons of accessory muscle have the potential to be used in the repair or replacement of damaged tendons through surgical transfer or transplantation [5].

The present case described an unusual combination of variations of the ECRB and ED muscles and discussed its embryological and clinical significances. Knowledge of anomalous variations of extensor tendons is important to the surgeon in order to avoid unintentional damage to healthy tendons during surgical procedure. Also, awareness of such anatomical variations is necessary for reconstructive surgeons and physicians for accurate restoration of 

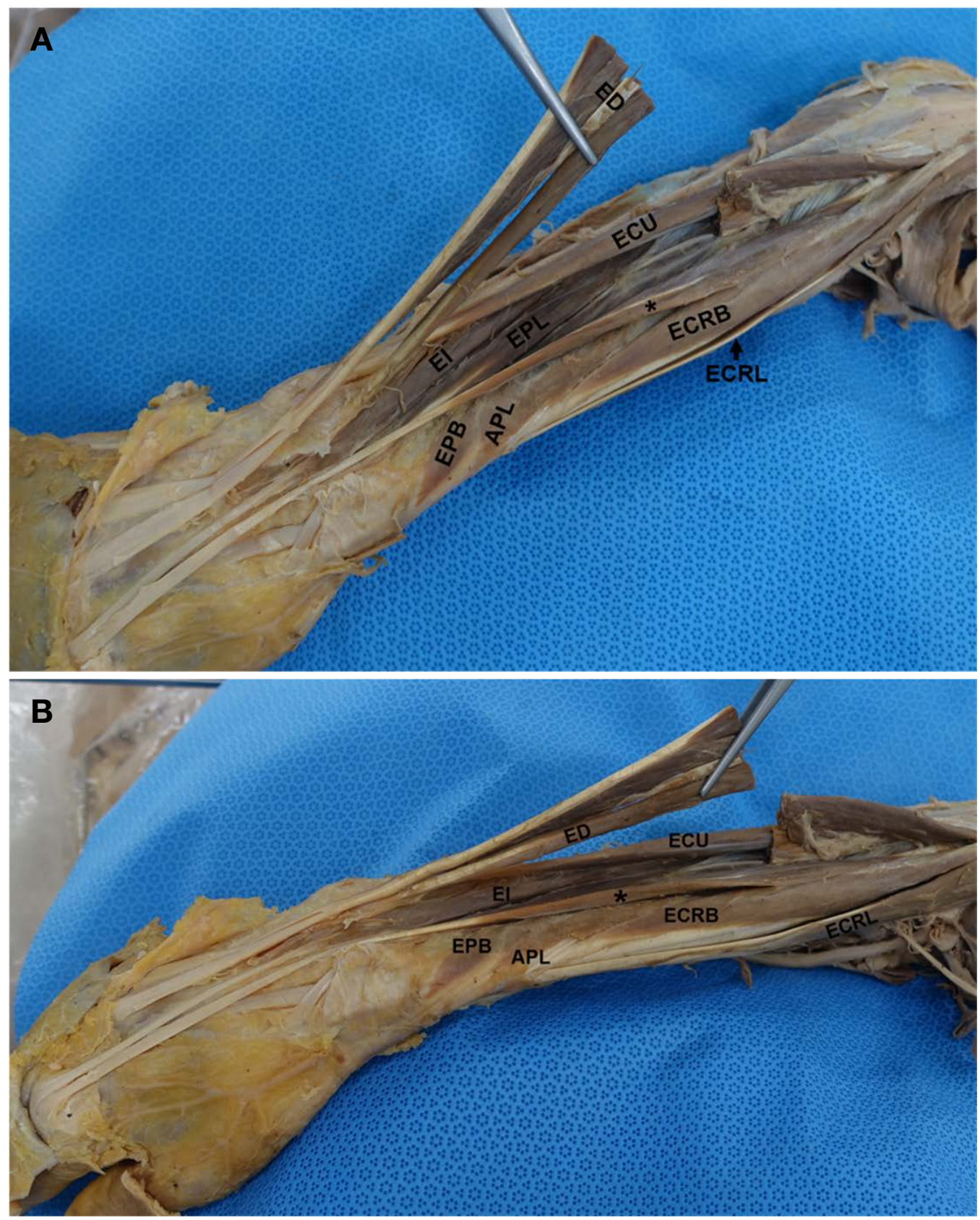

Fig. 1. Variations of the extensor muscles in right forearm. (A) Superior view. (B) Lateral view. *, accessory muscle of extensor carpi radialis brevis; APL, abductor pollicis longus; ECRB, extensor carpi radialis brevis; ECRL, extensor carpi radialis longus; ECU, extensor carpi ulnaris; ED, extensor digitorum; EI, extensor indicis; EPB, extensor pollicis bravis; EPL, extensor pollicis longus.

the hand function in cases of trauma and compression syndromes [1].

\section{CASE REPORT}

During a routine cadaveric dissection performed by un- dergraduate medical students, we observed an anomalous musculotendinous of the ECRB and ED muscle in the right upper limb of a cadaver of an 84-year-old Korean male (Fig. 1). After the skin, subcutaneous fat, and fascia, the extensor retinaculum was longitudinally opened to expose the extensor compartment muscles in the forearm. Each muscle was carefully observed, and the findings were photographed. In 
the extensor compartment of the forearm, after each extensor muscles were identified, the anomalous variation of the ECRB and ED muscles were found. Thin accessory muscle originated from the medial aspect of the ECRB muscle and inserted onto dorsum and proximal phalanx of the index finger. The accessory muscle separated from the ECRB at distance of $5.82 \mathrm{~cm}$ from the lateral epicondyle. Its main muscle belly measured approximately $11.3 \mathrm{~cm}$ in length and its musculotendinous portion measured approximately $13.6 \mathrm{~cm}$ in length. And its end measured approximately $3.84 \mathrm{~cm}$ in wide. In addition to this variation, the ED muscle originates from the lateral epicondyle of the humerus and its tendon divided into three distinct muscular tendons for middle, ring and little fingers distally. The index finger tendon of ED muscle was absent. There was no variation in the other neurovascular structures.

\section{DISCUSSION}

Anomalous muscles in the extensor compartment of the forearm are common. These variations are diagnosed incidentally, and it can be symptomatic. The anatomic variation of the extensor tendons of the forearm has been reported in the literature. Among these variations, the extensor carpi intermedius arises independently from the epicondyle of the humerus between the extensor carpi radialis longus (ECRL) and ECRB muscles, and inserts second or third metacarpal bone [6,7]. Other researchers reported that accessory muscle of the ECRB muscle originated from the ECRB muscle and inserted into dorsum of the index finger, and second metacarpal bone [8,9]. Arora et al. also reported an accessory muscle of ECRB for index finger and absence of the ED tendon for index finger [1]. And ED tendons for middle, ring and little fingers were multiple. Our case is similar with this case, however, ED tendons in present case was typical pattern. The origin and number of the tendon for index finger was variable [10], however, its origination from ECRB was exteremly rare. This case has not been matched any previous reports and is a first report in Korean cadaver.

In order to understand these variations, embryological knowledge is necessary. In the forearm, the precursor extensor muscle mass differentiates into a radial portion. Then, this radial portion divides into superficial and deep portions. Extensor digitorum, extensor carpi ulnaris, and extensor digiti minimi muscles are originated from the superficial portion while abductor pollicis longus, extensor pollicis brevis, extensor pollicis longus and extensor indicis muscles are originated from the deep portion $[11,12]$. Developmental defect in different stages of gestation is one of the reasons of the anatomical variations. Besides, various changes of extensor limb myotomes involved in the transformation of the somatic layer of lateral mesoderm; such as regression, retention, or reappearance may be the cause of variations of the tendons [13]. The embryological basis of these variations is explained by the fact that the precursor of extensor muscle may differentiate into three bundles namely the superficial extensor muscles and any developmental defect is related to the developing extensor sheet of the forearm [14].

Lateral epicondylitis or tennis elbow results from either acute strain or repetitive stresses to the origin of the ECRB muscle. It is also used for autologous blood injections for treatment of tennis elbow [15]. The ECRB muscle has gained importance for use in 'free functional muscle transfer i.e. transfer of a muscle with its motor nerve and vascular pedicle from one site of the body to another site in order to restore the motor function. The knowledge of the variations in the origin of its nerve supply is important while this muscle is being harvested Besides, the tendon of the extensor muscles is very superficial and is frequently injury than the tendons of flexor muscles [16]. Accessory tendons have the potential to be used in the repair or replacement of damaged tendons through surgical transfer or transplantation [5]. Anomalies of muscles and tendons in hand and wrist are significant during hand surgery. When found, at random during operation, these are often ignored, but it is sometimes responsible for different syndromes in and around the wrist. these may modify the maneuvers during tendon transfer or grafting [17]. The intersection syndrome (tenosynovitis of the radial wrist extensors) is associated with rubbing at the crossing point between the first dorsal compartment muscles and the radial wrist extensor muscles. These conditions can cause wrist and forearm pain, the most common are de Quervain tenosynovitis and thumb carp metacarpal arthritis [18]. High number of anatomical variations can cause de Quervain tenosynovitis [19]. These anatomical variations may lead to insufficient improvements even after tenosynovectomy. Knowledge of the existence of anomalous variations of the extensor tendon is important for radiologists, surgeons and anatomists to avoid surgical complications and prevent diagnostic errors. 


\section{REFERENCES}

1. Arora J, Dave V, Kumar A, Mehta V, Nayyar A, Suri RK, et al. Unusual architecture of extensor digitorum muscle of hand in conjunction with accessory belly of extensor carpi radialis brevis: a clinico-anatomical insight. Clin Ter. 2013;164:31-3.

2. Schenck RR. Variations of the Extensor Tendons of the Fingers. Surgical Significance. J Bone Joint Surg Am. 1964; 46:103-10

3. Das S, Sulaiman IM, Hussan F, Latiff AA, Suhaimi FH, Othman F. The additional tendons of the extensor digitorum muscle of the hand: an anatomical study with a clinical significance. Bratisl Lek Listy. 2008;109:584-6.

4. Shiraishi N, Matsumura G. Identification of two accessory muscle bundles with anomalous insertions in the flexor side of the right forearm. Okajimas Folia Anat Jpn. 2007;84:3542.

5. Bluth BE, Wu B, Elena Stark M, Wisco JJ. Variant of the extensor pollicis tertius: a case report on a unique extensor muscle to the thumb. Anat Sci Int. 2011;86:160-3.

6. Wood VE. The extensor carpi radialis intermedius tendon. J Hand Surg Am. 1988;13:242-5.

7. Albright JA, Linburg RM. Common variations of the radial writs extensors. J Hand Surg Am. 1978;3:134-8.

8. Melling M, Steindl M, Wilde J, Karimian-Teherani D. An anatomical variant of the extensor carpi radialis brevis muscle. Wien Klin Wochenschr. 2001;113:960-3.

9. Gümüşalan Y, Kalaycioğlu A, Yazar F, Arifoğlu Y, Sinav A. Accessory extensor carpi radialis muscle and interconnecting muscular bundle. Acta Anat (Basel). 1997;159:57-60.

10. von Schroeder HP, Botte MJ. Anatomy of the extensor ten- dons of the fingers; variations and multiplicity. J Hand Surg Am. 1995;20:27-34.

11. Yang K, Choi IJ, Lee JH. Accessory head of the extensor carpi radialis longus muscle merging with extensor carpi radialis brevis muscle. Surg Radiol Anat. 2018;40:1001-3.

12. Abdel-Hamid GA, El-Beshbishy RA, Abdel Aal IH. Anatomical variations of the hand extensors. Folia Morphol (Warsz). 2013;72:249-57.

13. Celik S, Bilge O, Pinar Y, Govsa F. The anatomical variations of the extensor tendons to the dorsum of the hand. Clin Anat. 2008;21:652-9.

14. Abu-Hijleh MF. Extensor pollicis tertius: an additional extensor muscle to the thumb. Plast Reconstr Surg. 1993;92:3403.

15. Edwards SG, Calandruccio JH. Autologous blood injections for refractory lateral epicondylitis. J Hand Surg Am. 2003;28:272-8.

16. Tuncali D, Yavuz N, Terzioglu A, Aslan G. The rate of upper-extremity deep-structure injuries through small penetrating lacerations. Ann Plast Surg. 2005;55:146-8.

17. Nayak SR, Krishnamurthy A, Prabhu LV, Jiji PJ, Ramanathan L, Kumar S. Multiple supernumerary muscles of the arm and its clinical significance. Bratisl Lek Listy. 2008;109:74-5.

18. Zhari B, Edderai M, Boumdine H, Amil T, En-nouali H. Dual intersection syndrome of the forearm: a case report. Pan Afr Med J. 2015;21:325.

19. Hoch J, Fritsch H, Lewejohann S. [Congenital or acquired disposition of the separate compartment of the extensor pollicis brevis tendon associated with stenosing tendovaginitis (de Quervain's disease)? Macroanatomical and fetal-plastinationhistological studies of the first compartment of the wrist]. Ann Anat. 2004;186:305-10. 